\title{
Oral care and oral assessment guide in breast cancer patients receiving everolimus and exemestane: subanalysis of a randomized controlled trial (Oral Care-BC)
}

\begin{abstract}
Masahiro Umeda ${ }^{1 \#}$, Yoshihide Ota ${ }^{2 \#}$, Kosuke Kashiwabara ${ }^{3}$, Naoki Hayashi ${ }^{4}$, Mariko Naito ${ }^{5}$, Toshinari Yamashita ${ }^{6}$, Hirofumi Mukai ${ }^{7}$, Katsuhiko Nakatsukasa ${ }^{8}$, Takeshi Amemiya ${ }^{9}$, Ken-Ichi Watanabe ${ }^{10}$, Hironobu Hata ${ }^{11}$, Yuichiro Kikawa ${ }^{12}$, Naoki Taniike ${ }^{13}$, Takashi Yamanaka ${ }^{6}$, Sachiyo Mitsunaga ${ }^{14}$, Kazuhiko Nakagami ${ }^{15}$, Moriyasu Adachi ${ }^{16}$, Naoto Kondo ${ }^{17}$, Yasuyuki Shibuya ${ }^{18}$, Naoki Niikura ${ }^{19}$

${ }^{1}$ Department of Clinical Oral Oncology, Nagasaki University Graduate School of Biomedical Sciences, Nagasaki, Japan; ${ }^{2}$ Department of Oral and Maxillofacial Surgery, Tokai University School of Medicine, Tokyo, Japan; ${ }^{3}$ Data Science Office, Clinical Research Promotion Center, The University of Tokyo Hospital, Tokyo, Japan; ${ }^{4}$ Department of Breast Surgical Oncology, St. Luke's International Hospital, Tokyo, Japan; ${ }^{5}$ Department of Oral Epidemiology, Graduate School of Biomedical and Health Sciences, Hiroshima University, Hiroshima, Japan; ${ }^{6}$ Department of Breast and Endocrine Surgery, Kanagawa Cancer Center, Kanagawa, Japan; ${ }^{7}$ Department of Breast and Medical Oncology, National Cancer Center Hospital East, Chiba, Japan; ${ }^{8}$ Department of Breast and Endocrine Surgery, Kyoto Prefectural University of Medicine, Kyoto, Japan; ${ }^{9}$ Department of Dentistry and Oral and Maxillofacial Surgery, Kyoto Prefectural University of Medicine, Kyoto, Japan; ${ }^{10}$ Department of Breast Surgery, Hokkaido Cancer Center, Hokkaido, Japan; ${ }^{11}$ Department of Dentistry and Oral Surgery, Hokkaido Cancer Center, Hokkaido, Japan; ${ }^{12}$ Department of Breast Surgery, Kobe City Medical Center General Hospital, Kobe, Japan; ${ }^{13}$ Department of Dentistry and Oral and Maxillofacial Surgery, Kobe City Medical Center General Hospital, Kobe, Japan; ${ }^{14}$ Department of Dentistry and Oral and Maxillofacial Surgery, Kanagawa Cancer Center, Kanagawa, Japan; ${ }^{15}$ Department of Breast and Endocrine Surgery, Shizuoka General Hospital, Shizuoka, Japan; ${ }^{16}$ Department of Oral and Maxillofacial Surgery, Shizuoka General Hospital, Shizuoka, Japan; ${ }^{17}$ Department of Breast and Endocrine Surgery, Nagoya City University Hospital, Nagoya, Japan; ${ }^{18}$ Department of Oral and Maxillofacial Surgery, Nagoya City University Graduate School of Medical Sciences, Nagoya, Japan; ${ }^{19}$ Department of Breast and Endocrine Surgery, Tokai University School of Medicine, Tokyo, Japan

Contributions: (I) Conception and design: All authors; (II) Administrative support: M Umeda, Y Ota, N Niikura; (III) Provision of study materials or patients: T Yamashita, T Yamanaka, K Nakatsukasa; (IV) Collection and assembly of data: S Mitsunaga, H Mukai, T Amemiya, H Hata, N Taniike, M Adachi, KI Watanabe, Y Kikawa, K Nakagami, N Kondo, Y Shibuya; (V) Data analysis and interpretation: K Kashiwabara, M Naito, N Hayashi; (VI) Manuscript writing: All authors; (VII) Final approval of manuscript: All authors.

"These authors contributed equally to this work.

Correspondence to: Masahiro Umeda. Department of Clinical Oral Oncology, Nagasaki University Graduate School of Biomedical Sciences, 1-7-1 Sakamoto, Nagasaki 852-8588, Japan. Email: mumeda@nagasaki-u.ac.jp.
\end{abstract}

Background: Oral mucositis is a clinically significant adverse event linked to cancer therapy; it reduces the quality of life of patients and may result in the discontinuation of treatment and a poorer prognosis. Based on level 3 evidence, the Mucositis Study Group of Multinational Association for Supportive Care in Cancer and the International Society of Oral Oncology recommend oral care for all patients receiving cancer chemotherapy and radiotherapy, although no data from large-scaled randomized controlled trials support the efficacy of oral care in preventing oral mucositis. Therefore, this randomized, controlled, multicenter, openlabel, phase III study sought to determine whether professional oral care reduces oral mucositis in everolimus and exemestane-treated estrogen receptor-positive metastatic breast cancer patients.

Methods: Altogether, 169 patients were randomized into the professional oral care $(n=82)$ and control $(n=87)$ groups. The professional oral care group received oral health instruction, professional mechanical tooth and tongue cleaning, gargling with a benzethonium chloride mouthwash, and dexamethasone ointment when grade 1 mucositis manifested. The control group received oral health instruction and gargling. Eight weeks after the everolimus and exemestane administration, the oral status (Oral Assessment Guide criteria) and oral mucositis status (Common Terminology Criteria for Adverse Events functional and clinical examinations) were evaluated. 
Results: The incidence of oral mucositis of any grade and grade 2 severe mucositis was significantly lower in the professional oral care group, based on the Common Terminology Criteria for Adverse Events functional and clinical examinations. The total Oral Assessment Guide score, total Oral Assessment Guide grade, and Oral Assessment Guide score of teeth/dentures and mucous membranes were significantly different between the two groups. The Oral Assessment Guide grade for swallow, lip, teeth/dentures, mucous membrane, tongue, and saliva significantly correlated to oral mucositis severity.

Conclusions: Professional oral care may prevent oral mucositis and improve teeth/denture conditions in patients receiving everolimus and exemestane.

Keywords: Benzethonium; breast neoplasm; dexamethasone; everolimus; exemestane

Submitted Sep 18, 2020. Accepted for publication Jan 08, 2021.

doi: 10.21037/atm-20-6488

View this article at: http://dx.doi.org/10.21037/atm-20-6488

\section{Introduction}

Oral mucositis is a clinically significant adverse event linked to cancer therapy. Its incidence ranges from 5\% to $40 \%$ among patients receiving standard-dose chemotherapy, and $75 \%$ or higher among patients receiving high-dose chemotherapy with stem-cell transplantation or radiation therapy for head and neck cancer $(1,2)$. When the oral cavity and the salivary glands are exposed to radiation therapy, hyposalivation, oral mucositis, loss of taste, trismus, radiation-induced dental caries, and osteoradionecrosis are the most common adverse events. Therefore, the early and active participation of dental professionals may be paramount in improving patients' quality of life during and after radiotherapy (3). Oral mucositis reduces the quality of life of patients and may result in the discontinuation of treatment and a poorer prognosis. Molecularly targeted therapeutic drugs can also cause oral mucositis. In particular, among patients receiving everolimus treatment, the incidence of oral mucositis of any grade is as high as $58 \%$ and is $81 \%$ among Asian patients and $91 \%$ among Japanese patients, based on subgroup analyses in the BOLERO-2 study (4,5). These findings could be because the recommended dose of everolimus $(10 \mathrm{mg})$ does not consider weight and body mass index, and Asian individuals have a smaller body surface area. Among Asians, people in East Asia, including Japan, are more likely to experience strong adverse effects because of their lower weight than that of people in West Asia and South Asia. However, the mechanisms and prevention of everolimus-induced oral mucositis have not been elucidated.

Based on level 3 evidence, the Mucositis Study Group of Multinational Association for Supportive Care in Cancer and the International Society of Oral Oncology (MASCC/ ISOO) recommend oral care for all patients receiving cancer chemotherapy and radiotherapy (2), although no data from large-scaled randomized controlled trials support the efficacy of oral care in preventing oral mucositis. Therefore, we conducted a randomized controlled trial to determine whether professional oral care (POC)—consisting of professional mechanical tooth cleaning, scaling, gargling with an antiseptic mouthwash containing benzethonium chloride, oral hygiene instruction, and use of dexamethasone ointment when grade 1 mucositis manifests-truly reduces oral mucositis in estrogen receptor-positive metastatic breast cancer patients treated with everolimus and exemestane [i.e., the Oral Care Evaluation to Prevent Oral Mucositis in Estrogen Receptor-Positive Metastatic Breast Cancer Patients Treated with Everolimus (Oral Care-BC) trial]. This subanalysis study focused on the relationship between POC and changes in the Oral Assessment Guide (OAG) score/grade $(6,7)$.

We present the following article in accordance with the CONSORT reporting checklist (available at http://dx.doi. org/10.21037/atm-20-6488).

\section{A summary of the study protocol and treatment in the Oral Care-BC trial}

Oral Care-BC is a Japan-based, phase 3, multicenter randomized clinical trial that assessed the effectiveness of POC in preventing oral mucositis in patients treated with everolimus and exemestane for hormone receptorpositive, human epidermal growth factor receptor type 2 (HER2)-negative metastatic breast cancer (8). Patients were 


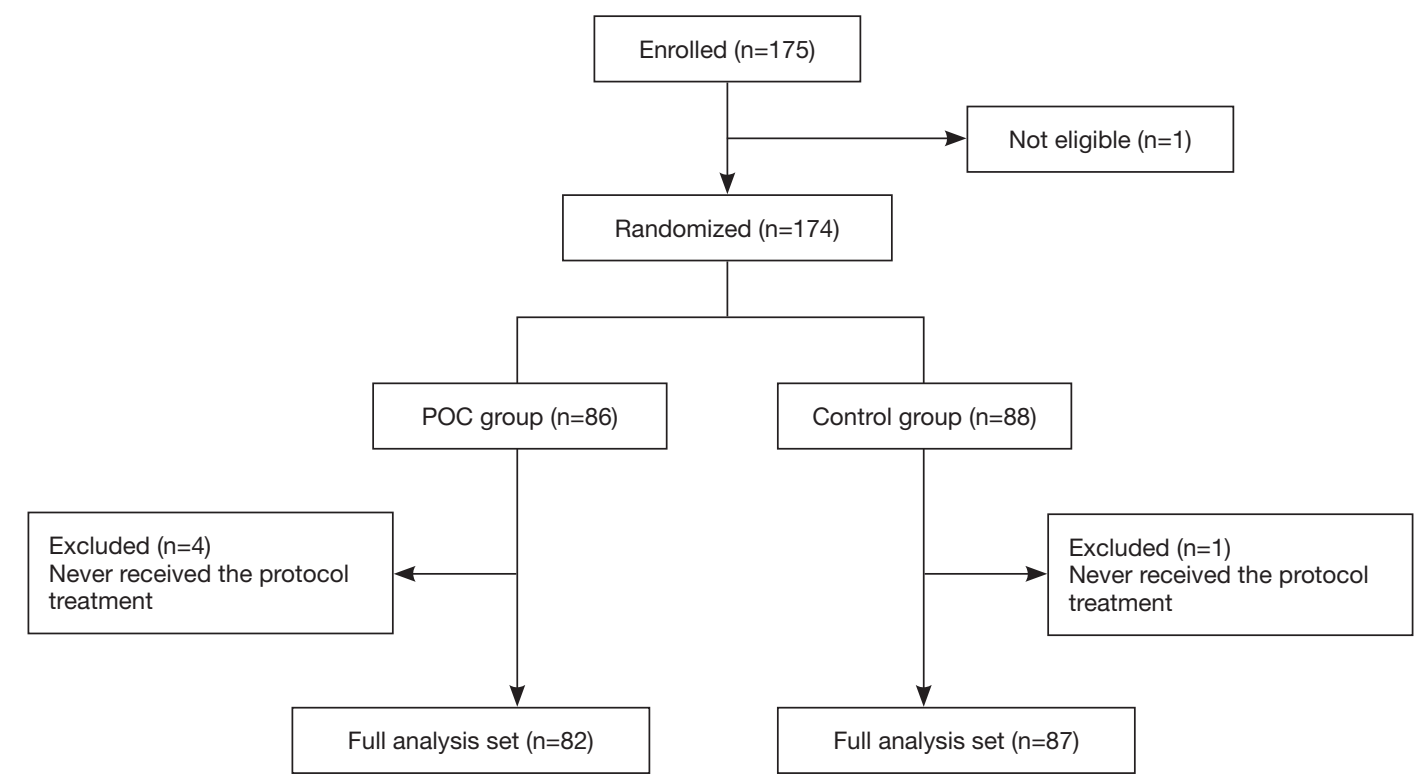

Figure 1 CONSORT diagram. POC, professional oral care; FAS, full analysis set; CONSORT, Consolidated Standards of Reporting Trials.

randomized into the POC group and control group in a 1:1 ratio (stratified, based on the center, use of bone-modifying agents, patient's age, and history of receiving chemotherapy within 3 months). Patients in the POC group received oral hygiene instruction, professional mechanical tooth cleaning, scaling, gargling with antiseptic mouthwash containing benzethonium chloride (Neostelin Green 0.2\% mouthwash solution; Nihon Shika Yakuhin, Co., Ltd., Osaka, Japan), and dexamethasone ointment (Dexaltin Oral Ointment, $1 \mathrm{mg} / \mathrm{g}$; Nihon Kayaku Co., Ltd., Tokyo Japan) when grade 1 mucositis manifested. The primary endpoint was the incidence of oral mucositis of any grade after everolimus and exemestane treatment, as evaluated with a functional examination.

The inclusion criteria were as follows: women aged $\geq 20$ years who were postmenopausal and had metastatic histologically or cytologically confirmed hormone receptorpositive, HER2-negative breast cancer; who were newly prescribed everolimus $10 \mathrm{mg}$ and exemestane $25 \mathrm{mg}$; had an Eastern Cooperative Oncology Group performance status of $0-1$ (9); and had adequate renal function (serum creatinine level $\leq 1.5 \times$ the upper limit of normal). The exclusion criteria were as follows: edentulism, oral mucositis within 1 month, chemotherapy administered within 1 month before randomization (except for bisphosphonate drugs or denosumab), and severe or uncontrolled medical conditions.

\section{Methods}

\section{Patients}

Patients treated with everolimus and exemestane for hormone receptor-positive, HER2-negative metastatic breast cancer were recruited in the study based on the aforementioned inclusion and exclusion criteria. Between May 2015 and December 2017, 175 patients were screened in Japan. Of these, 174 eligible patients from 31 centers were enrolled in the Oral Care-BC trial (Figure 1). Among the enrolled patients, 86 patients were allocated to the POC group and 88 patients were allocated to the control group. Four patients in the POC group and one in the control group were excluded from the study because they did not receive the protocol treatment. Thus, 169 patients (82 in the POC group and 87 in the control group) were examined.

\section{Ethical statement}

This study was conducted in accordance with the Declaration of Helsinki criteria (as revised in 2013) (10). Institutional review boards at each of the 31 study sites approved the study protocol. The registration number of each hospital was as follows: Aichi Cancer Center Central Hospital, 2014-1-163; Asahi University Murakami Memorial Hospital, 2015-3-11; Chiba University Hospital, 942; Gifu City Hospital, 312; Gunma Prefectural Cancer 
Center, 405-27017; Hamamatsu Medical Center, H273; Hamamatsu Oncology Center, 2015-001; Horoshima City Hospital, 26-128; Hokkaido Cancer Center, 14E15; Hyogo Medical University Hospital, 1924; Japanese Red Cross Society Nagasaki Atomic Bomb Hospital, 374; Kanagawa Prefectural Cancer Center, 27-22; Kawasaki Medical University Hospital, 2280; Kobe City Medical Center General Hospital, 14121; Kumamoto Morito General Hospital, 241-1; Kyoto Prefectural University Hospital, ERB-C-303; Nagasaki University Hospital, 15012606; Nagoya City University Hospital, 45-150015; National Cancer Center Hospital East, 2014-309; Nishigunma Hospital, 15-05-03; Osaka City University Hospital, 3203; Osaka Medical Center Hospital, 16221; Ota Memorial Hospital, 3203; Rinku General Medical Center, 551; Saitama Red Cross Hospital, 15-A; Sapporo Medical University Hospital, 272-12; Shikoku Cancer Center, 20154; Shizuoka General Hospital, 15-09-24; Teine Keijinkai Hospital, 27-4-20; Tokai University Hospital, 14R-063; Tokyo Metropolitan Cancer and Infectious Disease Center Komagome Hospital, 1550. Written informed consent was obtained from all participants included in the study.

\section{Evaluation of intraoral findings}

Before the administration of everolimus and exemestane, a dentist examined the oral status of each patient once weekly for 8 weeks using the OAG criteria, which were modified by Andersson et al. (6) from the original guideline described by Eilers (7). The OAG is used to evaluate the oral condition and is based on eight factors: swallow, lip, tongue, saliva, mucous membrane, gingivae, teeth/dentures, and voice. Each factor is categorized as grade 1 (normal), grade 2 (mild to moderate change), or grade 3 (moderate to severe change). Furthermore, the oral status is evaluated as "normal" when the total OAG score is $\leq 8$, "mild functional disturbance" when the total OAG score is 9-12, and "moderate or severe functional disturbance" when the total OAG score is $\geq 13$.

The patients were examined once weekly for oral mucositis by an oncologist, who used the functional examination of Common Terminology Criteria for Adverse Events (CTCAE) guidelines, version 4.0 (11), and by a dentist, who conducted clinical examinations in accordance with CTCAE guidelines, version 3.0 (12). The functional examination findings were "grade 1 " for asymptomatic or mild symptoms for which an intervention was not indicated; "grade 2" for moderate pain that did not interfere with oral intake but was an indication for a modified diet; and "grade 3" for severe pain that interfered with oral intake. The clinical examination findings were "grade 1" for erythema of the mucosa; "grade 2" for patchy ulcerations or pseudomembranes; and "grade 3" for confluent ulcerations or pseudomembranes, and bleeding with minor trauma. Before the administration of everolimus and exemestane, periodontal disease was further classified by a dentist into three categories: "mild" for a pocket depth $\leq 3.0 \mathrm{~mm}$ and mobility of grade 1 or less; "moderate" for a pocket depth of 4-6 mm or one or more teeth with grade 2 mobility; and "severe" for a pocket depth $\geq 7 \mathrm{~mm}$ or one or more teeth with grade 3 mobility.

\section{Outcomes}

The primary endpoint was to examine whether a difference would exist between the POC group and the control group in the total score of the OAG. The secondary endpoints were to examine whether (I) a difference existed in each OAG score between the POC group and the control group and (II) a relationship existed between oral mucositis and the total OAG score or between oral mucositis and each OAG score.

\section{Statistical analysis}

The differences in the incidence of oral mucositis in the POC group and control group were analyzed using Fisher's test. The differences in periodontal disease and the OAG score between the POC and control groups were analyzed using the chi-square test or the Wilcoxon rank-sum test. The longitudinal data of changes in the OAG score between the POC group and the control group were analyzed using the linear mixed effect model. A two-tailed $\mathrm{P}$ value of $5 \%$ or less was significant.

\section{Ethics and registration}

The study was approved by Institutional Review Board for Clinical Research, Tokai University (approval NO.: 14R063). The study protocol was registered on the website of the University Hospital Medical Information Network (Tokyo, Japan; protocol ID 000016109) on January 5, 2015, and registered on the ClinicalTrials.gov website (ClinicalTrials.gov identifier: NCT02376985) on March 3, 2015. Details are available at the following addresses:

(I) https://upload.umin.ac.jp/cgi-open-bin/ctr/ctr.cgi?fu 
Table 1 Characteristics of patients in the Oral Care-BC trial

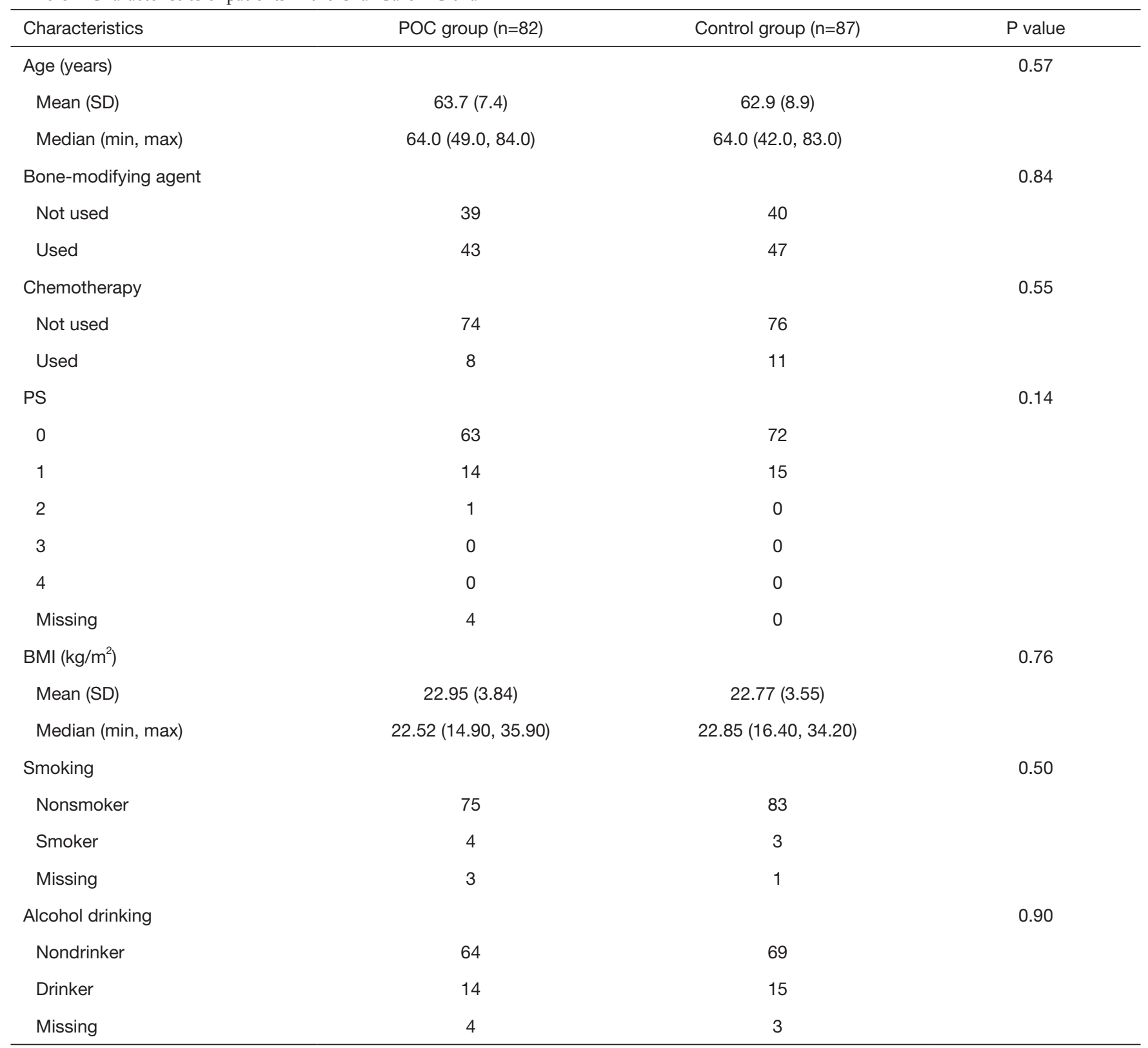

POC, professional oral care; SD, standard deviation; PS, performance status; BMI, body mass index.

nction $=$ brows $\&$ action $=$ brows $\&$ type $=$ summary $\&$ recpt no=R000018713\&language $=\mathrm{J}$;

(II) https://clinicaltrials.gov/ct2/show/NCT02376985.

\section{Results}

\section{Main Oral Care-BC trial results}

Tables 1,2 show patients' characteristics and main results of Oral Care-BC, as reported previously (11). Based on functional examinations, the incidence of grade 1 oral mucositis was significantly different between the POC group (75.6\%) and the control group (89.7\%) ( $\mathrm{P}=0.034)$. The incidence of grade 2 (i.e., severe) oral mucositis was also significantly different between the POC group (34.1\%) and the control group (54.0\%) $(\mathrm{P}=0.013)$. Based on clinical examinations, grade 1 oral mucositis occurred in $80.5 \%$ of 
Table 2 Incidence probability of oral mucositis

\begin{tabular}{|c|c|c|c|}
\hline Oral mucositis & POC group $(n=82)$ & Control group $(n=87)$ & $P$ value \\
\hline Yes & 62 & 78 & \\
\hline No & 20 & 9 & \\
\hline \multicolumn{4}{|l|}{ Grade 1 or more (by clinical examination) } \\
\hline No & 16 & 6 & \\
\hline \multicolumn{4}{|l|}{ Grade 2 or more (by functional examination) } \\
\hline Yes & 28 & 47 & 0.013 \\
\hline No & 54 & 40 & \\
\hline No & 49 & 26 & \\
\hline
\end{tabular}

POC, professional oral care.

the POC group patients and in $93.1 \%$ of the control group patients $(\mathrm{P}=0.034)$, and grade 2 oral mucositis occurred in $40.2 \%$ of the POC group patients and in $70.1 \%$ of the control group patients $(\mathrm{P}<0.001)$.

\section{Intraoral baseline findings in the POC and control groups}

Table 3 shows the baseline OAG score and grade, and periodontal disease status in the POC and control groups. Before the administration of everolimus and exemestane, the POC group patients had significantly more severe periodontal disease than the control group patients. The total OAG score, the OAG grade, and the OAG score of teeth/dentures were also worse in the POC group than in the control group.

\section{Change in the OAG score in the POC and control groups}

The total OAG score and grade in the control group increased in the first 2 weeks, whereas the total OAG score and grade in the POC group remained stable or slightly decreased throughout the study period. The longitudinal data of the total OAG score and total OAG grade were significantly different between the two groups (Figure 2). An examination of each OAG score revealed significant differences between the POC and control groups in the longitudinal data of teeth/dentures and mucous membranes
(Figure 3).

\section{Relationship between the OAG score and oral mucositis}

The relationship between the total OAG score/grade and oral mucositis, based on clinical and functional examinations, is presented in Table 4. A significant correlation existed in that the total OAG score and grade increased as oral mucositis became more severe. With regard to the relationship between each OAG grade and mucositis, the OAG grades for swallow, lip, teeth/dentures, mucous membranes, tongue, and saliva were significantly associated with the severity of oral mucositis (Table 5).

\section{Discussion}

In this randomized controlled trial, we examined whether POC would truly reduce oral mucositis in estrogen receptor-positive metastatic breast cancer patients treated with everolimus and exemestane. We found that the incidence of oral mucositis of any grade and severe mucositis of grade 2 was significantly lower in patients in the POC group than in the control group, and the grade of oral mucositis significantly correlated to OAG grade for swallow, lip, teeth/dentures, mucous membranes, tongue, and saliva. Oral mucositis is thought to be exacerbated by damage to the oral mucosa caused by the drug, secondary infection of 
Table 3 Background factors at baseline

\begin{tabular}{|c|c|c|c|}
\hline Factors & POC group ( $n=82$ ) & Control group $(\mathrm{n}=87)$ & $P$ value \\
\hline None & 18, $22.0(13.7-32.8)$ & $36,41.4$ (30.9-52.4) & \\
\hline Mild & $63,76.8(67.2-86.3)$ & $51,58.6(47.6-69.1)$ & \\
\hline Moderate & $0,0.0(0-4.5)$ & $0,0.0(0-4.2)$ & \\
\hline OAG (total score) & & & $<0.01^{\dagger}$ \\
\hline $\mathrm{N}$, mean (SD) & $80,9.4(1.6)$ & $87,8.9(1.4)$ & \\
\hline Median (min, max) & $9.0(8.0,14.0)$ & $8.0(8.0,15.0)$ & \\
\hline OAG (grade) & & & $0.01^{*}$ \\
\hline Voice & & & $0.33^{*}$ \\
\hline Grade 1 & $81,98.8(95.5-100.0)$ & 86, $98.9(93.8-100.0)$ & \\
\hline Grade 2 & $0,0.0(0-4.5)$ & $1,1.1(0-6.2)$ & \\
\hline Grade 3 & $0,0.0(0-4.5)$ & $0,0.0(0-4.2)$ & \\
\hline Swallowing & & & $-^{*}$ \\
\hline Grade 1 & $81,98.8$ (95.5-100.0) & $87,100.0(95.8-100.0)$ & \\
\hline Grade 2 & $0,0.0(0-4.5)$ & $0,0.0(0-4.2)$ & \\
\hline Tooth, denture & & & $<0.01^{*}$ \\
\hline Grade 1 & $41,50.0(39.3-61.9)$ & $63,72.4(61.8-81.5)$ & \\
\hline Grade 2 & 30, $36.6(26.6-48.5)$ & $22,25.3(16.6-35.7)$ & \\
\hline Grade 3 & $10,12.2(6.1-21.5)$ & $2,2.3(0.3-8.1)$ & \\
\hline Mucosa & & & $0.57^{*}$ \\
\hline Grade 1 & 73, $89.0(81.5-95.6)$ & $82,94.3(87.1-98.1)$ & \\
\hline Grade 2 & $7,8.5(3.5-17)$ & $4,4.6(1.3-11.4)$ & \\
\hline Grade 3 & $1,1.2(0-6.7)$ & $1,1.1(0-6.2)$ & \\
\hline Gingiva & & & $0.26^{*}$ \\
\hline Grade 1 & $48,58.5(47.8-70.1)$ & $62,71.3(60.6-80.5)$ & \\
\hline Grade 2 & $32,39.0(28.8-51.0)$ & 24, 27.6 (18.5-38.2) & \\
\hline Grade 3 & $1,1.2(0-6.7)$ & $1,1.1(0-6.2)$ & \\
\hline
\end{tabular}

Table 3 (continued) 
Table 3 (continued)

\begin{tabular}{lcc}
\hline Factors & POC group $(\mathrm{n}=82)$ & Control group $(\mathrm{n}=87)$ \\
\hline Tongue & & \\
Grade 1 & $74,90.2(83.0-96.5)$ & $84,93.1(85.6-97.4)$ \\
Grade 2 & $6,7.3(2.8-15.4)$ & $3,3.4(0.7-9.7)$ \\
Grade 3 & $1,1.2(0-6.7)$ & $0,0.0(0-4.2)$ \\
Xerostomia & & \\
Grade 1 & $70,85.4(77.0-93.0)$ & $81,93.1(85.6-97.4)$ \\
Grade 2 & $11,13.4(7.0-23.0)$ & $6,6.9(2.6-14.4)$ \\
Grade 3 & $0,0.0(0-4.5)$ & $0,0.0(0-4.2)$ \\
\hline
\end{tabular}

${ }^{\dagger}$, Wilcoxon rank sum test; *, Chi-square test. POC, professional oral care; Cl, confidence interval; OAG, Oral Assessment Guide; SD, standard deviation; BMI, body mass index.
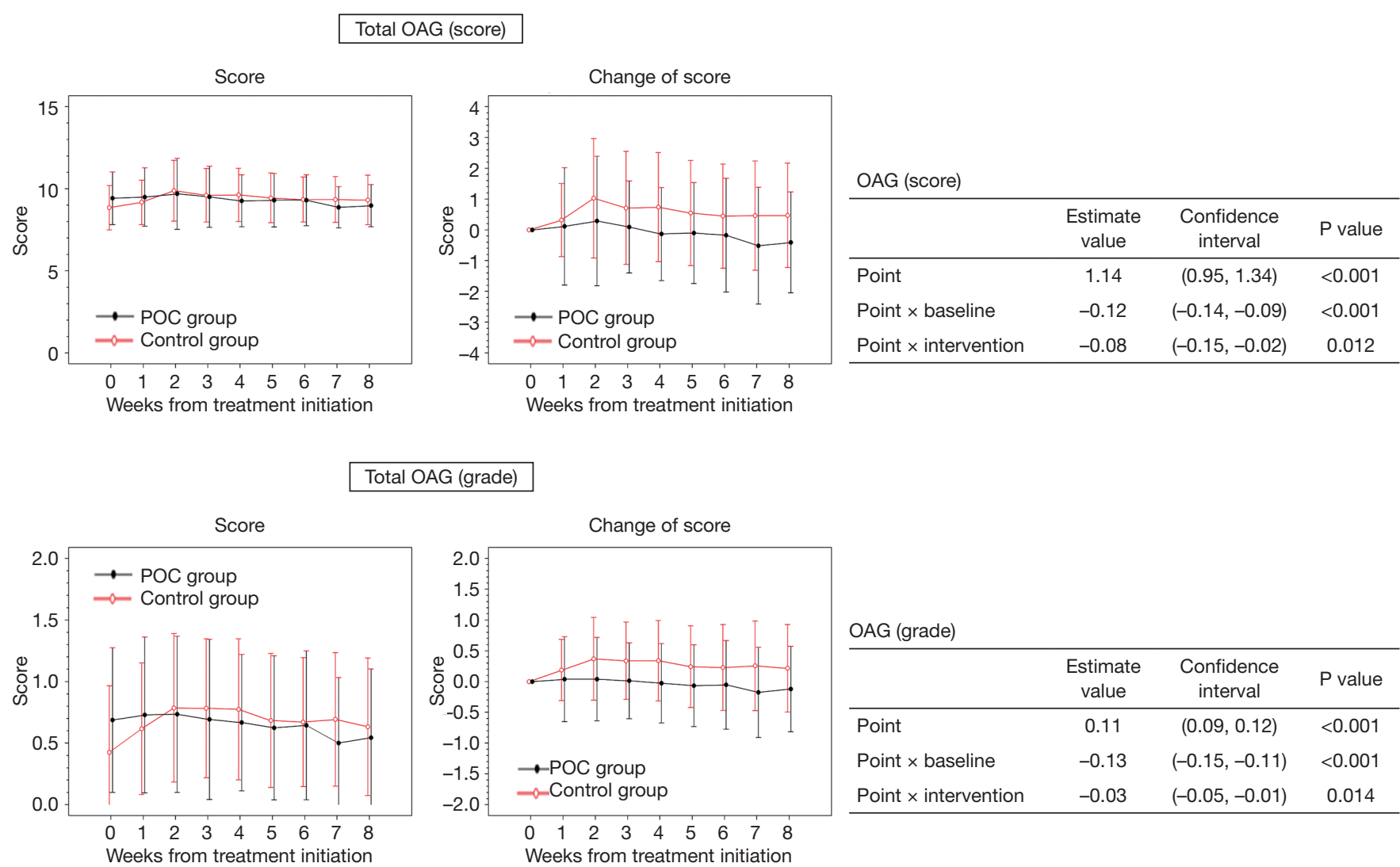

Figure 2 Longitudinal data representing the total OAG score/grade in the POC group and control group. OAG, Oral Assessment Guide; POC, professional oral care.

oral bacteria, and overproduction of cytokines. In the POC group, in addition to oral hygiene instruction, professional mechanical tooth cleaning, scaling, gargling with antiseptic mouthwash, and steroid ointment might suppress the secondary infection and cytokine overproduction, and prevent the aggravation of mucositis. However, the detailed mechanism by which POC inhibits the occurrence of mucositis or the increase in OAG score is unknown. In 

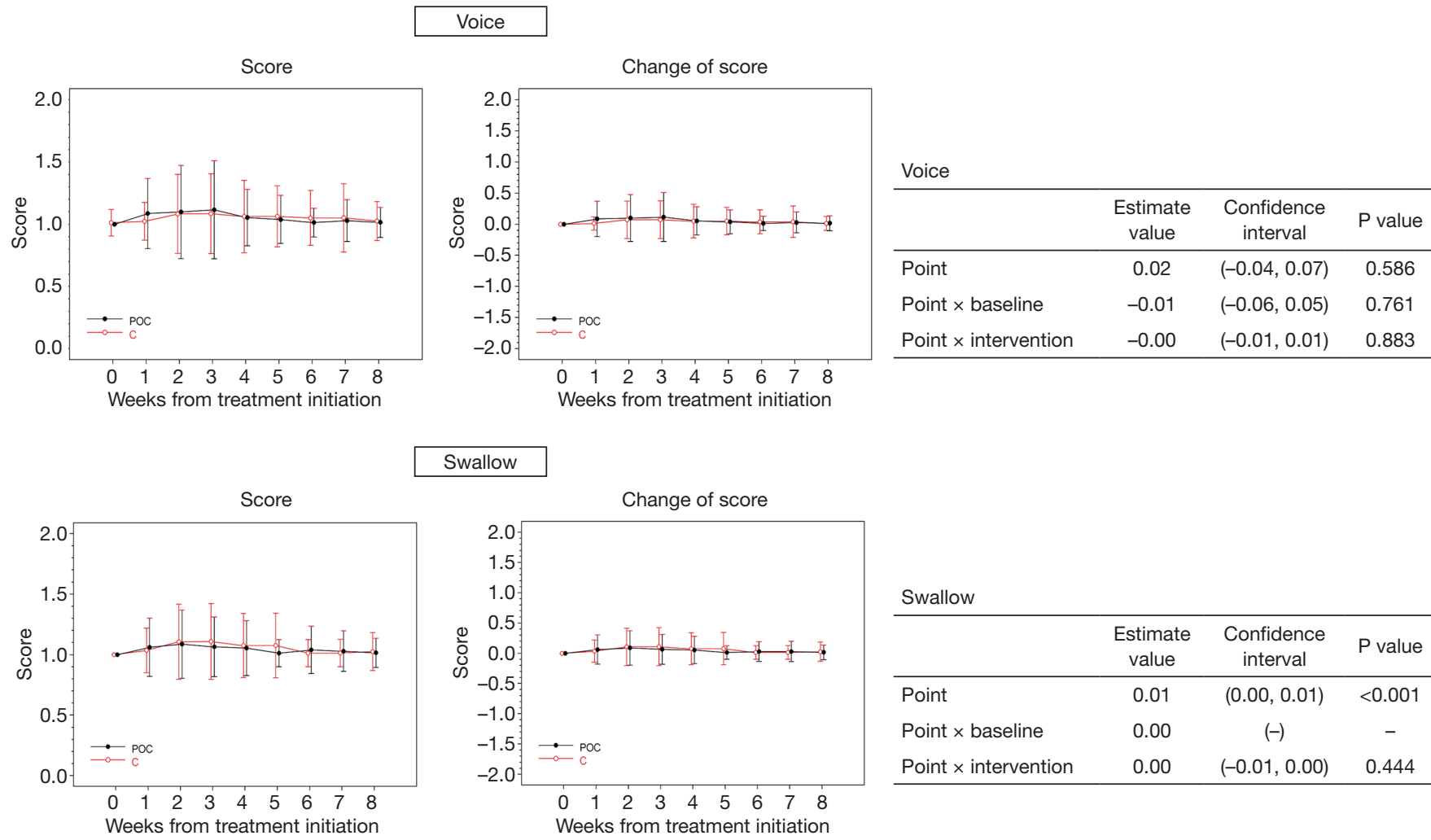

\section{Swallow}

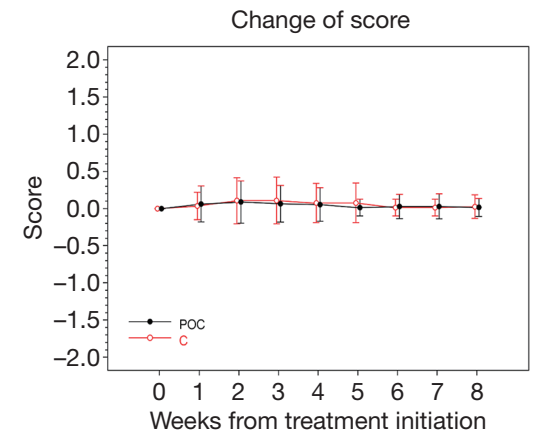

Swallow

\begin{tabular}{lccc}
\hline & $\begin{array}{c}\text { Estimate } \\
\text { value }\end{array}$ & $\begin{array}{c}\text { Confidence } \\
\text { interval }\end{array}$ & P value \\
\hline Point & 0.01 & $(0.00,0.01)$ & $<0.001$ \\
Point $\times$ baseline & 0.00 & $(-)$ & - \\
Point $\times$ intervention & 0.00 & $(-0.01,0.00)$ & 0.444 \\
\hline
\end{tabular}

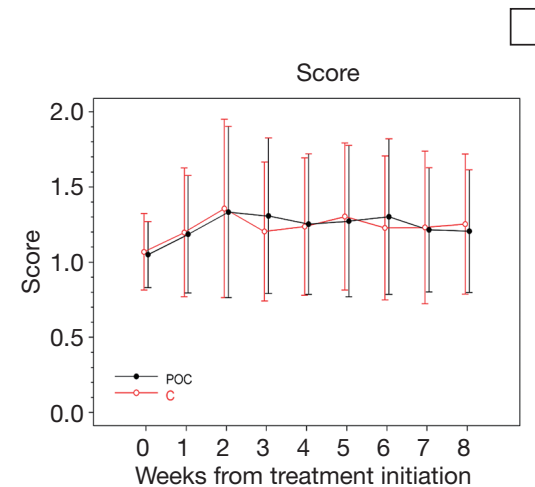

Lip

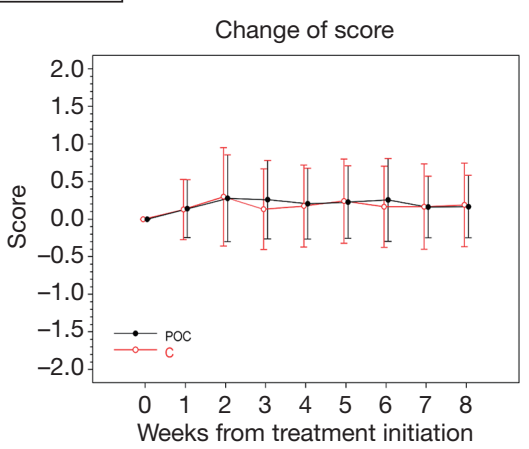

\begin{tabular}{lccc} 
Lip & $\begin{array}{c}\text { Estimate } \\
\text { value }\end{array}$ & $\begin{array}{c}\text { Confidence } \\
\text { interval }\end{array}$ & P value \\
\hline Point & 0.21 & $(0.16,0.26)$ & $<0.001$ \\
Point $\times$ baseline & -0.16 & $(-0.20,-0.12)$ & $<0.001$ \\
Point $\times$ intervention & 0.00 & $(-0.02,0.02)$ & 0.755 \\
\hline
\end{tabular}

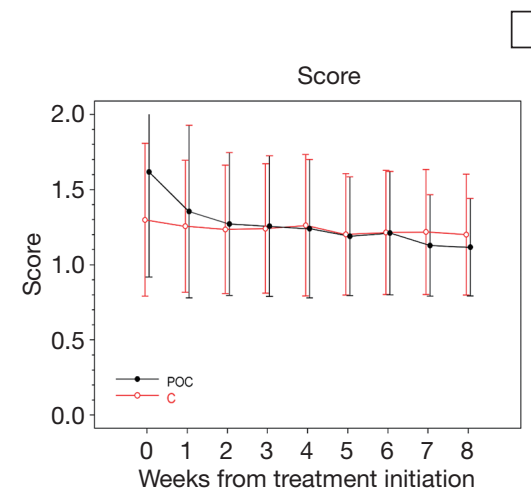

Teeth

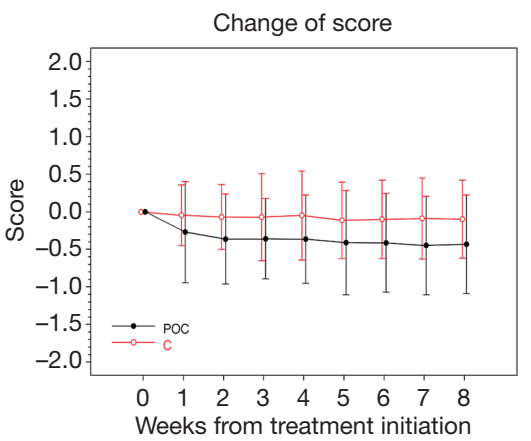

\begin{tabular}{lccc} 
Teeth & $\begin{array}{c}\text { Estimate } \\
\text { value }\end{array}$ & $\begin{array}{c}\text { Confidence } \\
\text { interval }\end{array}$ & P value \\
\hline Point & 0.16 & $(0.14,0.18)$ & $<0.001$ \\
Point $\times$ baseline & -0.13 & $(-0.15,-0.12)$ & $<0.001$ \\
Point $\times$ intervention & -0.02 & $(-0.04,0.00)$ & 0.026 \\
\hline
\end{tabular}

Figure 3 Longitudinal data representing each OAG score in the POC group and control group. OAG, Oral Assessment Guide; POC, professional oral care. 
Table 4 Relationship between OAG total score/grade and oral mucositis

\begin{tabular}{|c|c|c|c|c|c|c|c|c|c|c|c|c|}
\hline OAG & \multicolumn{6}{|c|}{ Grade of oral mucositis (by oncologist) } & \multicolumn{6}{|c|}{ Grade of oral mucositis (by dentist) } \\
\hline Total score & & & & & & $<0.01$ & & & & & & $<0.01$ \\
\hline 3 & 0 & 0 & 0 & 0 & 0 & & 0 & 0 & 0 & 0 & 0 & \\
\hline 4 & 0 & 0 & 0 & 0 & 0 & & 0 & 0 & 0 & 0 & 0 & \\
\hline 6 & 0 & 0 & 0 & 0 & 0 & & 0 & 0 & 0 & 0 & 0 & \\
\hline 7 & 0 & 0 & 0 & 0 & 0 & & 0 & 0 & 0 & 0 & 0 & \\
\hline 8 & 317 & 111 & 27 & 0 & 0 & & 494 & 110 & 34 & 1 & 0 & \\
\hline 9 & 195 & 97 & 28 & 4 & 0 & & 260 & 94 & 45 & 0 & 0 & \\
\hline 12 & 22 & 37 & 13 & 2 & 0 & & 38 & 25 & 21 & 4 & 0 & \\
\hline 13 & 12 & 17 & 13 & 1 & 0 & & 17 & 16 & 15 & 1 & 0 & \\
\hline 14 & 2 & 11 & 3 & 2 & 0 & & 6 & 9 & 8 & 0 & 0 & \\
\hline 15 & 0 & 2 & 0 & 2 & 0 & & 2 & 1 & 2 & 1 & 0 & \\
\hline 16 & 0 & 1 & 2 & 2 & 0 & & 0 & 1 & 4 & 0 & 0 & \\
\hline 17 & 1 & 0 & 0 & 0 & 0 & & 0 & 0 & 1 & 0 & 0 & \\
\hline 18 & 0 & 0 & 0 & 0 & 0 & & 0 & 0 & 0 & 0 & 0 & \\
\hline 19 & 0 & 0 & 1 & 0 & 0 & & 0 & 0 & 1 & 0 & 0 & \\
\hline 24 & 0 & 0 & 0 & 0 & 0 & & 0 & 0 & 0 & 0 & 0 & \\
\hline Total grade & & & & & & $<0.01$ & & & & & & $<0.01$ \\
\hline Normal & 317 & 111 & 27 & 0 & 0 & & 494 & 110 & 34 & 1 & 0 & \\
\hline Mild & 340 & 267 & 76 & 9 & 0 & & 482 & 215 & 153 & 5 & 0 & \\
\hline Moderate/severe & 15 & 31 & 19 & 7 & 0 & & 25 & 27 & 31 & 2 & 0 & \\
\hline
\end{tabular}

OAG, Oral Assessment Guide.

this study, the functional examination classification of oral mucositis was determined by an oncologist, and the clinical examination classification was determined by a dentist familiar with findings in the oral cavity. Similar results were obtained using any of the determination methods.

For patients with advanced breast cancer, the treatment aims to delay disease progression while minimizing treatment-related adverse events. Oral mucositis associated with mammalian target of rapamycin (mTOR) inhibitor drug use reduces the oral food intake, drug adherence, and quality of life of patients (13). Treatment with everolimus and exemestane includes various adverse events such as dermatitis, hyperglycemia, high cholesterol, infection, burning, interstitial pneumonia, thromboembolism, oral 
Table 5 Relationship between each OAG grade and oral mucositis

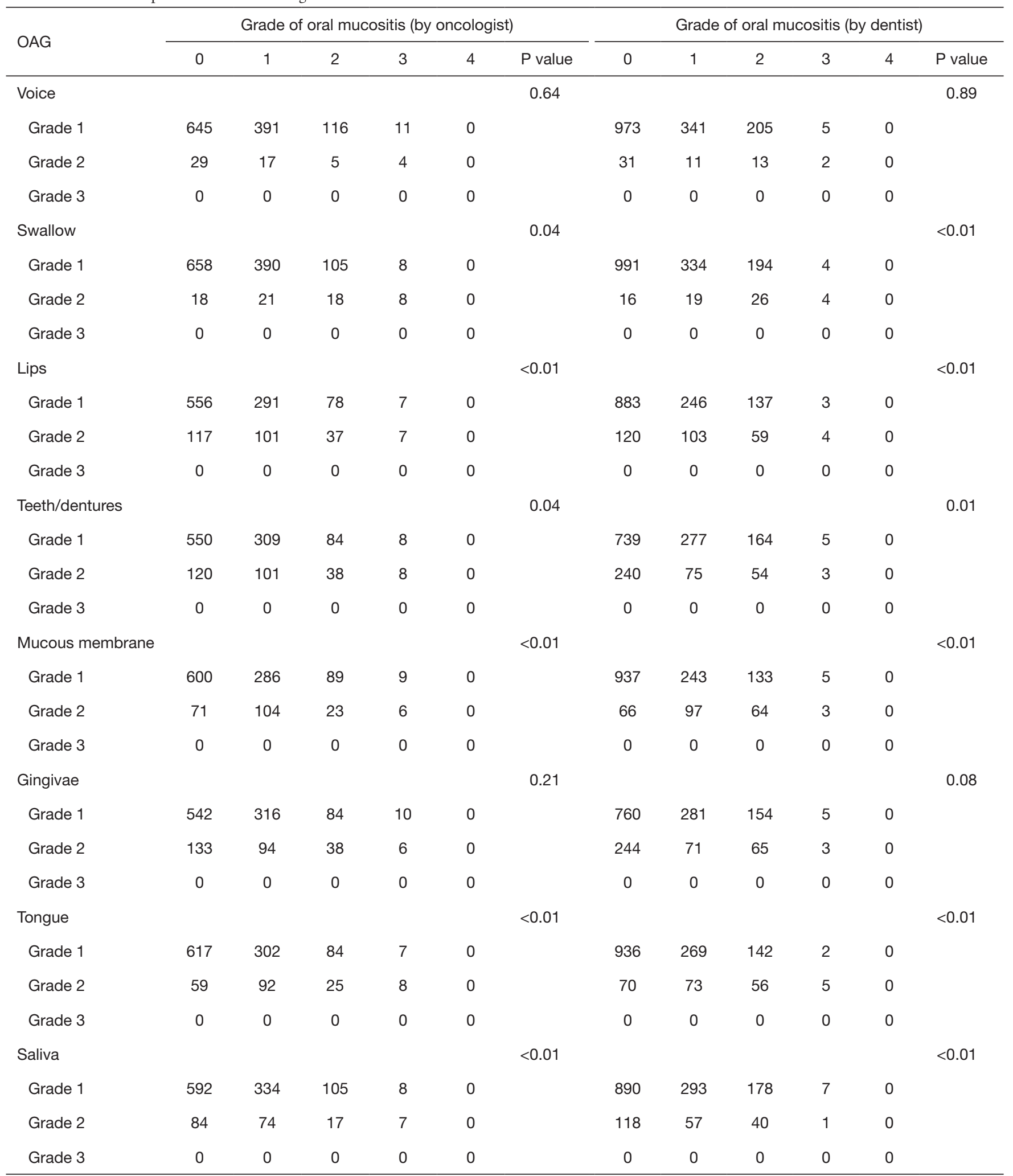

OAG, Oral Assessment Guide. 
mucositis, diarrhea, joint pain, osteoporosis, and eczema. Among them, the frequency of oral mucositis is relatively high, and when oral mucositis worsens to grade 2, the drug is often withdrawn. In this study, a total of 24 out of 169 patients were temporarily withdrawn, most of the reasons being oral mucositis. On account of the clinical benefits and potential long-term use of everolimus and exemestane, establishing an effective strategy is desirable to prevent and reduce oral mucositis associated with these drugs.

Rugo et al. (14) reported that the prophylactic use of dexamethasone mouthwash reduced the incidence of severe oral mucositis in women with hormone receptorpositive, HER2-negative metastatic breast cancer (i.e., the SWISH trial). However, their study was a single-arm, phase 2 trial with a historical control. Since steroid-containing mouthwashes have not been approved in Japan, we decided to investigate the effectiveness of steroid ointments in this study. Similar to their study, our results also show the effectiveness of topical steroids for oral mucositis.

To the best of our knowledge, the Oral Care-BC trial is the first randomized controlled trial that has clarified the efficacy of POC in preventing chemotherapy-related oral mucositis. Furthermore, the use of mouthwash containing steroids has not been allowed in Japanese insurance practice, and only dexamethasone- or triamcinolone-containing ointments have been approved for use on the oral mucosa.

Several assessment methods of the oral cavity have been used during chemotherapy or radiotherapy. Among these methods, Eilers OAG (7) is the most relevant method used in daily clinical practice (15). The revised OAG is a revision of Eilers' OAG that can be applied to young people and to the elderly (7). The relationship between the use of everolimus and exemestane and the change in the OAG score/grade have not been reported. Our study revealed that the OAG score increased in the control group, whereas it did not increase in the POC group, and that the OAG score increased because of an increase in the scores for mucous membranes and teeth/dentures. However, other scores for voice, swallow, lip, gingivae, tongue, and saliva were not increased in either group.

With regard to the relationship between oral mucositis and the OAG score, the severity of mucositis was associated with the total OAG score and with the score of mucous membranes and swallow, lip, tongue, and saliva. These findings suggest that POC can prevent oral mucositis and improve the condition of teeth/dentures in patients receiving everolimus and exemestane treatment, and that preventing severe oral mucositis may improve swallow, lip, tongue, and saliva scores, although no significant difference existed in the current study.

This study has some limitations. First, our study was an open-label study with a small number of patients. Thus, the possibility of bias in the assessments cannot be denied. Second, the control group consisted of patients who did not receive POC and who were not intended of being treated with a steroid mouthwash, which is standard in some countries, because steroid mouthwash products are not available in Japan. Third, detailed data on oral hygiene and periodontal disease in both groups were not investigated in this study. Therefore, the mechanism by which POC suppresses the incidence of mucositis or the increase in the OAG score could not be fully elucidated.

In conclusion, the Oral Care-BC trial showed that POC is effective in suppressing oral mucositis in breast cancer patients receiving everolimus and exemestane therapy (16). Efficient oral care can be easily administered globally and should be considered a new standard prior to the administration of these drugs, especially in the first 8 weeks of treatment. Furthermore, POC may also be helpful in reducing oral mucositis when using everolimus for other diseases such as renal cell carcinoma, subependymal giant cell astrocytoma associated with tuberous sclerosis complex, and advanced neuroendocrine tumors.

\section{Acknowledgments}

We greatly appreciate all the women who participated in this trial. We also thank all investigators and their collaborators who were dedicated to this study. We thank Editage (www.editage.jp) for their English-language editing services.

Funding: This work was supported by Comprehensive Support Project for Oncology Research (CSPOR) of the Public Health Research Foundation. The research fund was provided to CSPOR by Novartis Pharma K. K. (Tokyo, Japan). Novartis Pharma K. K. took no part in this study, other than providing information relevant to the proper use of the study drug. All decisions concerning the planning, implementation, and publication of this study were made by the steering committee.

\section{Footnote}

Reporting Checklist: The authors have completed the CONSORT reporting checklist. Available at http://dx.doi. org/10.21037/atm-20-6488 
Data Sharing Statement: Available at http://dx.doi. org/10.21037/atm-20-6488

Conflicts of Interest: All authors have completed the ICMJE uniform disclosure form (available at http://dx.doi. org/10.21037/atm-20-6488). Dr. NH reports personal fees from Chugai-Pharma, Novartis, AstraZeneca, Devicor Japan, and Allegan Japan, outside the submitted work. Dr. TY reports grants and other from Chugai, other from Eisai, other from Novartis Pharma, grants and other from Taiho, other from Astrazeneca, grants and other from Kyowa Kirin, other from Pfizer Japan, other from Eli Lilly, outside the submitted work. Dr. HM received honoraria from AstraZeneca, Pfizer, Takeda, Daiichi Sankyo and Taiho, and research grant from Japanese government, Daiichi Sankyo, Eisai, Nippon Kayaku and Pfizer, outside the submitted work. Dr. YK reports personal fees from Eisai, personal fees from Chugai, personal fees from Novartis, personal fees from Taiho, personal fees from Pfizer, personal fees from Eli Lilly, outside the submitted work. Dr. NN reports grants from Novartis, Bristol-Myers Squibb, Chugai Pharmaceutical Co., Nihon Medi-Physics Co., MSD, and Daiichi-Sankyo, personal fees from AstraZeneca, Novartis, Eisai, and Pfizer, outside the submitted work. The other authors have no conflicts of interest to declare.

Ethical Statement: The authors are accountable for all aspects of the work in ensuring that questions related to the accuracy or integrity of any part of the work are appropriately investigated and resolved. This study was conducted in accordance with the Declaration of Helsinki criteria (as revised in 2013). Institutional review boards at each of the 31 study sites approved the study protocol. The registration number of each hospital was as follows: Aichi Cancer Center Central Hospital: 2014-1-163, Asahi University Murakami Memorial Hospital: 2015-3-11, Chiba University Hospital: 942, Gifu City Hospital: 312, Gunma Prefectural Cancer Center: 405-27017, Hamamatsu Medical Center: H27-3, Hamamatsu Oncology Center: 2015-001, Horoshima City Hospital: 26-128, Hokkaido Cancer Center: 14E15, Hyogo Medical University Hospital: 1924, Japanese Red Cross Society Nagasaki Atomic Bomb Hospital: 374, Kanagawa Prefectural Cancer Center: $27-$ 22, Kawasaki Medical University Hospital: 2280, Kobe City Medical Center General Hospital: 14121, Kumamoto Morito General Hospital: 241-1, Kyoto Prefectural University Hospital: ERB-C-303, Nagasaki University Hospital: 15012606, Nagoya City University Hospital: 45-
15-0015, National Cancer Center Hospital East: 2014-309, Nishigunma Hospital: 15-05-03, Osaka City University Hospital: 3203, Osaka Medical Center Hospital: 16221, Ota Memorial Hospital: 3203, Rinku General Medical Center: 551, Saitama Red Cross Hospital: 15-A, Sapporo Medical University Hospital: 272-12, Shikoku Cancer Center: 20154, Shizuoka General Hospital: 15-09-24, Teine Keijinkai Hospital: 27-4-20, Tokai University Hospital: 14R-063, Tokyo Metropolitan Cancer and Infectious Disease Center Komagome Hospital: 1550. Informed consent was taken from all individual participants.

Open Access Statement: This is an Open Access article distributed in accordance with the Creative Commons Attribution-NonCommercial-NoDerivs 4.0 International License (CC BY-NC-ND 4.0), which permits the noncommercial replication and distribution of the article with the strict proviso that no changes or edits are made and the original work is properly cited (including links to both the formal publication through the relevant DOI and the license). See: https://creativecommons.org/licenses/by-nc-nd/4.0/.

\section{References}

1. Sonis ST, Elting LS, Keefe D, et al. Perspectives on cancer therapy-induced mucosal injury: pathogenesis, measurement, epidemiology, and consequences for patients. Cancer 2004;100:1995-2025.

2. Lalla RV, Bowen J, Barasch A, et al. MASCC/ISOO clinical practice guidelines for the management of mucositis secondary to cancer therapy. Cancer 2014;120:1453-61. Erratum in: Cancer. 2015 Apr 15;121(8):1339.

3. Kielbassa AM, Hinkelbein W, Hellwig E, et al. Radiationrelated damage to dentition. Lancet Oncol 2006;7:326-35.

4. Noguchi S, Masuda N, Iwata H, et al. Efficacy of everolimus with exemestane versus exemestane alone in Asian patients with HER2-negative, hormone-receptorpositive breast cancer in BOLERO-2. Breast Cancer 2014;21:703-14.

5. Ito $\mathrm{Y}$, Masuda $\mathrm{N}$, Iwata $\mathrm{H}$, et al. Everolimus plus exemestane in postmenopausal patients with estrogenreceptor-positive advanced breast cancer - Japanese subgroup analysis of BOLERO -2. Gan To Kagaku Ryoho 2015;42:67-75.

6. Andersson P, Hallberg IR, Renvert S. Inter-rater reliability of an oral assessment guide for elderly patients residing in a rehabilitation ward. Spec Care Dentist 2002;22:181-6.

7. Eilers J, Berger AM, Petersen MC. Development, testing, 
and application of the oral assessment guide. Oncol Nurs Forum 1988;15:325-30.

8. Niikura N, Ota Y, Hayashi N, et al. Evaluation of oral care to prevent oral mucositis in estrogen receptor-positive metastatic breast cancer patients treated with everolimus (Oral Care-BC): randomized controlled phase III trial. Jpn J Clin Oncol 2016;46:879-82.

9. ECOG-ACRIN Cancer Research Group. ECOG performance status. 2019. Available online: https://ecogacrin.org/resources/ecog-performance-status. Accessed 20 April 2019.

10. World Medical Association. World Medical Association Declaration of Helsinki: ethical principles for medical research involving human subjects. JAMA 2013;310:2191-4.

11. National Cancer Institute. Common Terminology Criteria for Adverse Events (CTCAE) version 4.0. 2009. Available online: https://evs.nci.nih.gov/ftp1/ CTCAE/CTCAE_4.03/CTCAE_4.03_2010-06-14_ QuickReference_5x7.pdf. Accessed 20 April 2020.

12. National Cancer Institute. Common Terminology Criteria

Cite this article as: Umeda $\mathrm{M}$, Ota $\mathrm{Y}$, Kashiwabara K, Hayashi N, Naito M, Yamashita T, Mukai H, Nakatsukasa K, Amemiya T, Watanabe KI, Hata H, Kikawa Y, Taniike N, Yamanaka T, Mitsunaga S, Nakagami K, Adachi M, Kondo N, Shibuya Y, Niikura N. Oral care and oral assessment guide in breast cancer patients receiving everolimus and exemestane: subanalysis of a randomized controlled trial (Oral Care-BC). Ann Transl Med 2021;9(7):535. doi: 10.21037/atm-20-6488 for Adverse Events v3.0 (CTCAE). 2006. Available online: https://ctep.cancer.gov/protocolDevelopment/electronic_ applications/docs/ctcaev3.pdf. Accessed 20 April 2020.

13. Rugo HS, Hortobagyi GN, Yao J, et al. Meta-analysis of stomatitis in clinical studies of everolimus: incidence and relationship with efficacy. Ann Oncol 2016;27:519-25.

14. Rugo HS, Seneviratne L, Beck JT, et al. Prevention of everolimus-related stomatitis in women with hormone receptor-positive, HER2-negative metastatic breast cancer using dexamethasone mouthwash (SWISH): a single-arm, phase 2 trial. Lancet Oncol 2017;18:654-62.

15. Glenny AM, Gibson F, Auld E, et al. The development of evidence-based guidelines on mouth care for children, teenagers and young adults treated for cancer. Eur J Cancer 2010;46:1399-412.

16. Niikura N, Nakatukasa K, Amemiya T, et al. Oral Care Evaluation to Prevent Oral Mucositis in Estrogen Receptor-Positive Metastatic Breast Cancer Patients Treated with Everolimus (Oral Care-BC): A Randomized Controlled Phase III Trial. Oncologist 2020;25:e223-30. 